\title{
Vibrational dynamics of plant light-harvesting complex LHC II investigated by quasi- and inelastic neutron scattering
}

\author{
Maksym Golub ${ }^{1}$, Klaus-Dieter Irrgang ${ }^{2}$, Leonid Rusevich ${ }^{3}$ and Jörg Pieper ${ }^{1, \text { a }}$ \\ ${ }^{1}$ Tartu University, Institute of Physics, Tartu, Estonia \\ ${ }^{2}$ Department of Life Science \& Technology, Laboratory of Biochemistry, University for Applied Sciences, Berlin, \\ Germany \\ ${ }^{3}$ Institute of Physical Energetics, Riga, Latvia
}

\begin{abstract}
Vibrational dynamics of the light-harvesting complex II (LHC II) from spinach was investigated by quasi- and inelastic neutron scattering (QENS and INS) at three different temperatures of 80, 160, and $285 \mathrm{~K}$. QENS/INS spectra of solubilised LHC II and of the corresponding buffer solution were obtained separately and exhibit characteristic inelastic features. After subtraction of the buffer contribution, the INS spectrum of LHC II reveals a distinct Boson peak at $\sim 2.5 \mathrm{meV}$ at $80 \mathrm{~K}$ that shifts towards lower energies if the temperature is increased to $285 \mathrm{~K}$. This effect is interpreted in terms of a "softening" of the protein matrix along with the dynamical transition at $\sim 240 \mathrm{~K}$. Our findings indicate that INS is a valuable method to obtain the density of vibrational states not only at cryogenic, but also at physiological temperatures.
\end{abstract}

\section{Introduction}

Photosynthetic antenna (or "light-harvesting") complexes are designed to absorb solar energy in a broad spectral range and to efficiently transfer the resulting excitation energy to reaction center complexes, where the primary charge separation and further electron transfer takes place (for a review see e.g. van Amerongen \& Croce [1]). The major antenna complex of green plants is LHC II, which accounts for approximately $65 \%$ of all chlorophyll associated with Photosystem II. Its structure is well characterized by X-ray crystallography up to atomic resolution [2,3]. According to these studies each LHC II monomer binds 14 chlorophyll and 4 carotenoid molecules for efficient light-harvesting. The protein matrix of LHC II is formed by three membrane spanning and two amphipathic $\alpha$-helices located at the lumenal side of the thylakoid membrane $[2,3]$.

The ultrafast excitation energy transfer (EET) processes in antenna complexes are mediated via the coupling of electronic transitions of pigment molecules to lowfrequency vibrations of the protein matrix, also referred to as electron-phonon coupling (for a review see [4]). So far, electron-phonon coupling has been mainly investigated by high-resolution optical spectroscopies at low temperatures, which are referred to as spectral hole burning (SHB) and fluorescence line-narrowing (FLN) (for a review see [4]). The electron-phonon coupling strengths reported for antenna complexes are often weak or moderate with coupling constants (Huang-Rhys factors S) in the range of $0.5-1.5$. The one-phonon profiles observed are typically strongly asymmetric with peak energies at about $2.5 \mathrm{meV}$ (see e.g. [4] and references therein). However,

\footnotetext{
${ }^{a}$ Corresponding author: pieper@ut.ee
}

experimental techniques like SHB and FLN are technically restricted to temperatures below about $40 \mathrm{~K}$ [4], because these spectrally selective methods require a static (frozen) ensemble of protein conformations. As a result, electronphonon coupling could not be investigated at temperatures higher than accessible by SHB and FLN. Therefore, an independent experimental approach is required to study the vibrational density of states of photosynthetic pigmentprotein complexes at elevated or even physiological temperatures.

In this regard, QENS and INS are valuable experimental tools for direct studies of vibrational and conformational protein dynamics (for reviews see $[5,6]$ ). However, so far QENS results are available only for intact Photosystem II membranes containing LHC II [7, 8], but not for isolated antenna complexes. Therefore, QENS spectra of solubilised LHC II trimers and of the corresponding buffer solution were measured at temperatures of 80,160 and $285 \mathrm{~K}$ in order to characterize the functionally important one-phonon profiles of the pigment-protein complex.

\section{Materials and methods}

For this experiment solubilized LHC II was produced from Photosystem II membrane fragments from spinach by sucrose density gradient centrifugation according to the procedure previously described in the literature [9]. Furthermore, $\mathrm{H}_{2} \mathrm{O}$ contained in the buffer solution was replaced by $\mathrm{D}_{2} \mathrm{O}$ following the method described in [10] in order to reduce the scattering contribution of the solvent. The LHC II content in the solubilized sample was determined independently to be about $300 \mathrm{mg}$ in a total volume of $2 \mathrm{ml}$ according to the procedure described in [10]. Furthermore, it was checked by extensive

This is an Open Access article distributed under the terms of the Creative Commons Attribution License 4.0, which permits unrestricted use, distribution, and reproduction in any medium, provided the original work is properly cited. 
biochemical analyses prior to and after the neutron scattering experiment that the sample remained intact in its native trimeric form [10] preventing aggregation [11] and monomerization [12].

QENS/INS experiments were performed using the spectrometer NEAT (Helmholtz Zentrum Berlin, Germany). The spectra were recorded using an incident neutron wavelength of $5.1 \AA$. The elastic resolution of $0.117 \mathrm{meV}$ was determined by vanadium runs. QENS/INS measurements of LHC II and of the $\mathrm{D}_{2} \mathrm{O}$ containing buffer were carried out separately in order to quantify the contribution of the solvent. The QENS/INS data were normalized, corrected for detector efficiency and sample geometry dependent attenuation, transferred to energy scale, and summed over all scattering angles using the program package FITMO.

In the case of a protonated scatterer like a protein or a biological membrane, it is sufficient to consider only the incoherent contribution to the double-differential cross section, so that

$$
\frac{\delta^{2} \sigma}{\delta \Omega \delta \omega}=\frac{\left|\kappa_{1}\right|}{\left|\kappa_{0}\right|} b_{i n c}^{2} S_{i n c}(Q, \omega) .
$$

In this expression, $k_{0}$ and $k_{1}$ are the wave vectors of the incident and scattered neutrons, respectively, $\mathrm{b}_{\text {inc }}$ is the incoherent scattering length, $\mathrm{S}_{\text {inc }}(Q, \omega)$ is the incoherent scattering function with $Q$ being the momentum transfer defined by $Q=k_{1}-k_{0}$, and $\hbar \omega$ reflects the neutron energy. In practice, $S_{\text {inc }}$ is not directly accessible by measurements and has to be replaced by the experimental scattering function $S_{\text {exp }}$, which is given by $[5,6]$

$$
\begin{aligned}
& S_{\exp }(Q, \omega)= \\
& F_{N} \exp \left\{-\frac{h \omega}{2 k T}\right\} R(Q, \omega) \otimes S_{\text {theo }}(Q, \omega) .
\end{aligned}
$$

In this equation, $\mathrm{F}_{\mathrm{N}}$ is a normalization factor, $\exp \left\{-\frac{h \omega}{2 k T}\right\}$ the detailed balance factor with $k$ being the Boltzmann constant and $T$ the temperature, $\mathrm{R}(Q, \omega)$ the experimentally obtained resolution function, and $\mathrm{S}_{\text {theo }}(Q, \omega)$ the theoretical model function describing the dynamics of the sample. The latter theoretical scattering function can be generally described by the following phenomenological model function $[5,6]$ :

$$
\begin{aligned}
& S_{\text {theo }}(Q, \omega)=\exp \left[-\langle u\rangle^{2} Q^{2}\right] * \\
& *\left\{A_{0}(Q) \delta(\omega)+\sum_{n} A_{n}(Q) L_{n}\left(H_{n}, \omega\right)+S_{\text {in }}(Q, \omega)\right\}
\end{aligned}
$$

which consists of three main contributions: i) a $\delta(\omega)-$ shaped elastic component, ii) a sum of quasielastic Lorentzian-shaped components $\mathrm{L}_{n}\left(\mathrm{H}_{n}, \omega\right)$ with half-width at half maximum (HWHM) $\mathrm{H}_{\mathrm{n}}$, and iii) an inelastic part $\mathrm{S}_{\text {in }}(Q, \omega)$ describing low-frequency vibrational motions. The fractional intensities of the elastic and quasielastic contributions are given by the elastic and quasielastic incoherent structure factors (EISF and QISF), $\mathrm{A}_{0}(Q)$ and $\mathrm{A}_{\mathrm{n}}(Q)$, respectively. The term $e^{-\left\langle u^{2}\right\rangle Q^{2}}$ is the DebyeWaller factor characterized by the vibrational mean square displacement $\left\langle\mathrm{u}^{2}\right\rangle$.

The QENS spectra were fitted with a model scattering function comprising a Gaussian resolution function

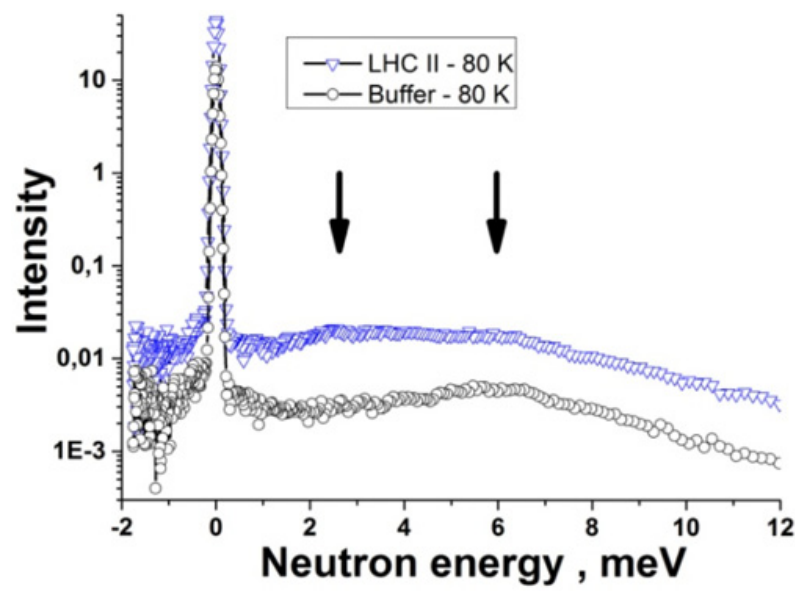

Figure 1. Comparison of the QENS spectra of solubilized LHC II (blue circles) and buffer solution (black circles) measured at $80 \mathrm{~K}$ and summed over all scattering angles resulting in $Q=1.46 \AA^{-1}$.

$\mathrm{R}(Q, \omega)$ and a Lorentzian quasielastic component with a width (FWHM) of $507 \mu \mathrm{eV}$. Since the Lorentzian width and the QISF are correlated parameters in purely phenomenological fits at one resolution, the width was kept constant when determining the temperature dependence of the relative quasielastic intensity in terms of the QISF.

To fit the inelastic part of the spectra the empirical pulse function

$$
S_{\text {in }}=c_{1} * c_{2} *\left(1-\exp \left[-\frac{w-x_{o}}{t_{1}}\right]\right) * \exp \left[-\frac{w-x_{o}}{t_{2}}\right]
$$

has been used, where $\mathrm{x}_{0}$ is the peak position and $\mathrm{t}_{1}$ and $\mathrm{t}_{2}$ are the widths describing the asymmetric shape. In addition, two pre-factors were applied: i) the so called DHO pre-factor [13]

$$
c_{1}=\left(\exp \left[\left\langle u^{2}\right\rangle Q^{2}\right]-1\right) .
$$

which was kept constant for all temperatures assuming a small variation of $\left\langle u^{2}\right\rangle[10]$ and ii) the temperature prefactor that can be described as [13]

$$
c_{2}=\frac{\omega}{1-\exp \left[-\frac{\omega}{k T}\right]} .
$$

This model function allowed us to precisely follow the changes in the shape of the Boson peak including its width and its peak position as a function of temperature.

\section{Results}

Before analyzing the quasi- and inelastic data of LHC II, it is necessary to properly subtract the scattering contribution of the solvent to the QENS spectra of the solubilized sample. QENS spectra of solubilized trimeric LHC II and buffer solution measured at $80 \mathrm{~K}$ are shown in Fig. 1. It is apparent that the QENS spectrum of solubilized LHC II exhibits two peaks at about 2.5 and $7 \mathrm{meV}$, respectively. The latter peak is also visible in the buffer spectrum, so that this feature can be clearly attributed to the solvent. At the same time, it is obvious that the buffer contribution is 


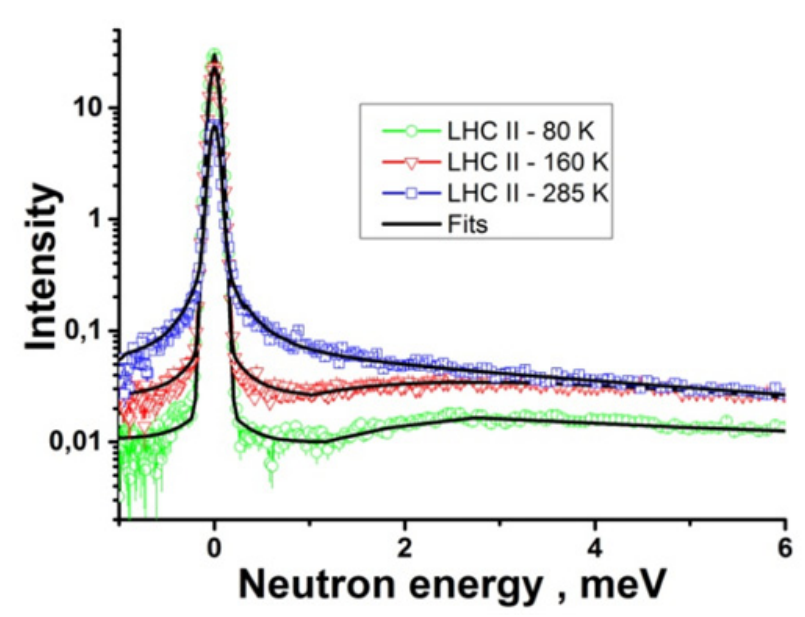

Figure 2. A) Representative QENS spectra of LHC II as obtained after subtraction of the buffer contribution at temperatures of $80 \mathrm{~K}$ (green circles), $160 \mathrm{~K}$ (red triangles), $285 \mathrm{~K}$ (blue squares), and fits according to Eqs. (3) and (5) (black lines). The spectra are summed over all scattering angles resulting in $Q=1.46 \AA^{-1}$.

less pronounced in the low-frequency region at about 2$3 \mathrm{meV}$. The buffer spectrum was subtracted from that of the solubilized sample according to its weight contribution. Note in this regard that the LHC II content can be determined independently via absorbance measurements according to the procedure described in [10]. The resulting difference curves reflecting the scattering functions $S(Q, w)$ of LHC II at different temperatures are shown in Fig. 2. As expected, the $80 \mathrm{~K}$ difference spectrum exhibits a Boson peak with a maximum at about $2-3 \mathrm{meV}$ and a widely asymmetric lineshape similar to the low-temperature results reported in [10]. It was also shown previously that the Boson peak in LHC II follows the typical Qdependence expected for harmonic vibrations [10]. At higher temperatures, however, the peak appears to shift to smaller energies, but is overlapping with the emerging quasielastic contribution.

According to our analysis (see examples of the fit results in Fig. 2), QENS spectra measured at temperatures below $\sim 80 \mathrm{~K}$ can be successfully fitted by an elastic peak only, which is represented by a resolution-broadened Gaussian function, i.e. a quasielastic contribution to the INS spectra is not observed in this case. This finding indicates that conformational dynamics is absent and the protein is trapped in individual conformational substates.

For temperatures higher than $\sim 80 \mathrm{~K}$ it is necessary to take into account a quasielastic contribution by a Lorentzian having a width (FWHM) of $507 \mu \mathrm{eV}$ in order to obtain a reasonable fit of the QENS spectra (see Fig. 2). As seen in Fig. 3, the resulting QISFs calculated from the fits show a linear increase in the temperature region between 80 and $240 \mathrm{~K}$ indicating the activation of reorientational motions of methyl groups and H-bonds $[14,15]$. Above $240 \mathrm{~K}$, the QISFs obtained from the fits are increasing significantly with increasing temperature. Such a behavior is characteristic for the dynamical transition corresponding to the onset of stochastic reorientational motions [5,6,14-17]. Similar results were obtained by QENS measurements of PS II membrane fragments [18].

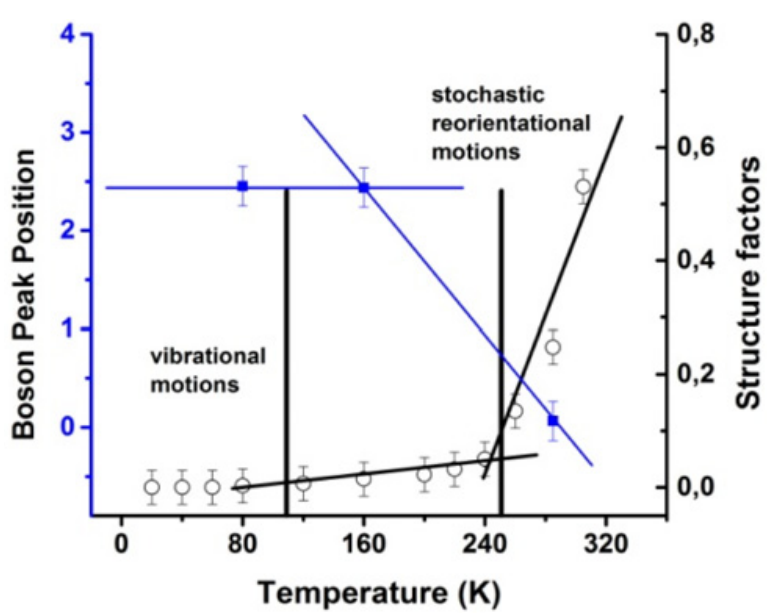

Figure 3. Temperature dependence of the QISFs obtained from the fits of the QENS spectra shown in Fig. 2 (black circles) and peak position of the Boson peak (blue squares). The full lines are given for ease of inspection.

Based on the latter study, it is possible to infer that the dynamical transition in PS II and its constituents like LHC II is influenced by the environment, since this effect is hydration-dependent $[8,18,19]$.

Finally, we discuss the temperature dependence of the Boson peak determined from the fits shown in Fig. 2. As visible Fig. 3, the peak position of the Boson peak reveals a pronounced shift to lower energies with increasing temperature above $240 \mathrm{~K}$. This effect is different from the low-temperature behavior of LHC II, where the density of vibrational states remained virtually identical below $\sim 120 \mathrm{~K}[10]$. The same is regularly assumed in simulations of EET, which are often restricted to the case of low (non-physiological) temperatures (see [4] and references therein). The Boson peak shift may be rationalized assuming that the softening of the protein matrix at the dynamical transition leads to a shift of the normal modes to lower energies, but further detailed studies are necessary to corroborate this correlation. Similar effects were reported previously for a polymer glass [20], for other proteins [21,22], and more recently for intact PS II membrane fragments [19]. However, to the best of our knowledge, it is the first indication of such a phenomenon for a photosynthetic antenna complex like LHC II. As a consequence, simulations of EET kinetics in lightharvesting complexes at physiological temperatures should account for a temperature-dependent density of vibrational states.

\section{Conclusions}

In the present study, QENS/INS experiments are applied to investigate the protein vibrations of the LHC II antenna complex. The temperature dependence of the QISFs reveals that the dynamical transition in LHC II occurs at about $240 \mathrm{~K}$. INS data show the presence of a Boson peak with a maximum at about $2.5 \mathrm{meV}$ at $80 \mathrm{~K}$, which is shifting towards lower energies upon temperature increase. One possible interpretation is that a softening of the protein matrix leads to generally lower 
vibrational eigenfrequencies at elevated temperatures. In antenna complexes, the coupling of low-frequency protein vibrations to electronic transitions of pigment molecules assures directed EET between different pigment molecules and to the reaction center (for a review see [4]). Therefore, a proper characterization of LHC II protein vibrations by INS is a prerequisite for a deeper understanding of EET at physiological temperatures.

We thank for support by European Social Fund's Internationalisation Programme DoRa, the Estonian Research Council (Grants ETF 9453, IUT 2-28 and SLOKT 12026 T) and by HZB Berlin, Germany. L. R. and J. P. are grateful to the exchange program between the Estonian and the Latvian Academies of Sciences for travel support. We are also grateful to S. Kussin and M. Weß (TU Berlin) for help in sample preparation.

\section{References}

[1] H. van Amerongen, R. Croce, in Primary processes of photosynthesis; Renger, G., Ed.; RSC Publ. Cambridge, U.K. I, 329 (2008)

[2] Z. Liu, H. Yan, K. Wang, T. Kuang, J. Zhang, L. Gui, X. An, W. Chang, Nature 428, 287 (2004)

[3] J. Standfuss, M. Lamborghini, W. Kühlbrandt, A. C. T. van Scheltinga, EMBO J. 24, 919 (2005)

[4] R. Jankowiak, M. Reppert, V. Zazubovich, J. Pieper, T. Reinot, Chem. Rev. 111, 4546 (2011)

[5] F. Gabel, D. Bicout, U. Lehnert, M. Tehei, M. Weik, G. Zaccai Q. Rev. Biophys. 35, 327 (2002)

[6] J. Pieper, G. Renger, Photosynth. Res. 102, 281 (2009)
[7] P. Kühn, J. Pieper, O. Kaminskaya, H.-J. Eckert, R. E. Lechner et al., Photosyn. Res. 84, 317 (2005)

[8] J. Pieper, T. Hauß, A. Buchsteiner, G. Renger, Eur. Biophys. J. 37, 657 (2008)

[9] K.-D Irrgang, E. J. Boekema, J. Vater, G. Renger, Eur. J. Biochem. 178, 209 (1988)

[10] J. Pieper, K-D. Irrgang, G. Renger, R.E. Lechner, J. Phys. Chem. B 108, 10556 (2004)

[11] J. Pieper, K.-D. Irrgang, M. Rätsep, R. Jankowiak, T. Schrötter, G. J. Small, J. Voigt, G. Renger, J. Phys. Chem. B 103, 2422 (1999)

[12] J. Pieper, M. Rätsep, K.-D. Irrgang, A. Freiberg, J. Phys. Chem. B 113, 10870 (2009)

[13] Lechner, R. E.; Riekel, C. in Neutron Scattering and Muon Spin Rotation, Springer Verlag, Berlin, Heidelberg, New York, Tokyo, p. 23 (1983)

[14] J. E. Curtis, M. Tarek, D. J. Tobias J. Am. Chem. Soc. 126, 15928 (2004)

[15] J. H. Roh, V. N. Novikov, R. B. Gregory, J. E. Curtis et al., Phys Rev Lett 95, 038101 (2005)

[16] J. Fitter, R.E. Lechner, N.A. Dencher, J. Phys. Chem. B 103, 8036 (1999)

[17] W. Doster, Eur. Biophys. J. 37, 591 (2008)

[18] J. Pieper, T. Hauß, A. Buchsteiner, K. Baczynski et al., Biochemistry 46, 11398 (2007)

[19] J. Pieper, M. Trapp, A. Skomorokhov, I. Natkaniec, J. Peters, G. Renger, Biochim. Biophys. Acta 1817, 2013 (2012)

[20] B. Frick, D. Richter, Phys Rev B 47, 14795 (1993)

[21] H. Nakagawa, Y. Joti, A. Kitao, M. Kataoka, Biophys. J. 95, 2916 (2008)

[22] A. Orecchini, A. Paciaroni, A. R. Bizzarri, S. Cannistraro, J. Phys. Chem. B 105, 12150 (2001) 